\title{
MiRNA-335-5p negatively regulates granulosa cell proliferation via SGK3 in PCOS
}

\author{
Lihua $\mathrm{Yao}^{1,2}$, Mingyang $\mathrm{Li}^{1,2}$, Jingwen $\mathrm{Hu}^{1,2}$, Wangsheng Wang ${ }^{1,2}$ and Minzhi Gao ${ }^{1,2}$ \\ ${ }^{1}$ Center for Reproductive Medicine, Renji Hospital, School of Medicine, Shanghai Jiao Tong University, Shanghai, \\ China and 'Shanghai Key Laboratory for Assisted Reproduction and Reproductive Genetics, Shanghai, China
}

Correspondence should be addressed to M Gao; Email: 328gao@sina.com

\begin{abstract}
Polycystic ovary syndrome (PCOS) is a major cause of infertility in women of reproductive age. However, its exact etiology remains unknown. In this study, we sequenced miRNAs in human follicular fluid and identified 16 downregulated and 3 upregulated miRNAs in PCOS group compared with non-PCOS group. Among the differential expressed miRNAs, miR-335-5p was verified lower abundance in PCOS than non-PCOS group using quantitative real-time PCR. Besides, miR-335-5p negatively correlated with antral follicle count, anti-Müllerian hormone and total testosterone. Bioinformatics analysis identified serum/glucocorticoid-regulated kinase family member 3 (SGK3) as a potential target gene of miR-335-5p. SGK3 is involved in protein kinase B-mammalian target of rapamycin kinase (AKT-mTOR) signaling pathway and cell proliferation. Western blotting and cell counting kit-8 assays demonstrated that miR-335-5p mimic reduced, while miR-335-5p inhibitor increased, SGK3 abundance, AKT-mTOR pathway and cell proliferation in human granulosa-like tumor KGN cells. Dual-luciferase reporter assays showed that miR-335-5p binds to the 3' untranslated region of SGK3 mRNA. Furthermore, miR-335-5p was decreased and SGK3 was elevated in human granulosa cells obtained from PCOS patients as compared with non-PCOS controls. These findings suggested that miR-335-5p is involved in granulosa cells proliferation by reducing SGK3 expression, which might provide a molecular target to improve dysfunctional granulosa cells in patients with PCOS.

Reproduction (2018) 156 439-449
\end{abstract}

\section{Introduction}

Polycystic ovary syndrome (PCOS) is a clinical syndrome involving metabolic abnormalities and reproductive disorders in reproductive aged women. It is one of the most common endocrine disorder, with an approximate incidence of 5-10\% (Goodarzi et al. 2011). The characteristics of PCOS are hyperandrogenemia, polycystic ovarian morphology, menstrual dysfunction, chronic oligo-anovulation and metabolic complications (Moran \& Teede 2009, Goodarzi et al. 2011). Many studies have suggested that the etiology of PCOS is related to genetic and environmental factors; however, little is known about its exact etiology.

Dysfunction of folliculogenesis is a leading cause of infertility in PCOS (Das et al. 2008. Franks et al. 2008, Patel \& Carr 2008). Human follicular fluid not only provides a life-sustaining microenvironment for oocytes and surrounding granulosa cells but also contains many important regulatory molecules that are important for the maturation and quality of oocytes (Hanrieder et al. 2008, Revelli et al. 2009). Therefore, follicular fluid plays a crucial role in folliculogenesis. In addition to secretions of granulosa and thecal cells, follicular fluid also contains many plasma components that cross the blood-follicular barrier via follicular basal lamina and thecal capillaries (Rodgers \& Irving-Rodgers 2010), including proteins, metabolites and miRNAs (Hanrieder et al. 2008, 2009, Revelli et al. 2009, Sang et al. 2013).

miRNAs are single-stranded non-coding endogenous RNAs (approximately 20-24 nucleotides in length), which can bind to target gene mRNA, particularly at the $3^{\prime}$ untranslated region ( $3^{\prime}$ UTR), thereby regulating its expression (Bartel 2009, Shukla et al. 2011). miRNAs are shown to play vital roles in a wide range of physiological processes at the post-transcriptional level (Ying et al. 2006). Aberrant expression of miRNAs has been found to accelerate cell proliferation in tumorigenesis and to regulate pathological pathways (Johnnidis et al. 2008, Ganepola et al. 2014, Huan et al. 2016). miRNAs can play important roles in the female reproductive system and folliculogenesis (Carletti \& Christenson 2009). Sang et al. first reported that the supernatant, microvesicles or exosomes from cell-free human follicular fluid contain miRNAs, which are important in metabolic and reproductive signaling pathways (Sang et al. 2013). Moreover, the miRNAs in human follicular fluid are powerful tools in the in vitro fertilization (IVF) process, which can predict the development of expanded blastocysts and clinical pregnancy outcome (Scalici 
et al. 2016). However, there have been few studies concerning the relationship between miRNAs in human follicular fluid and the pathogenesis of PCOS. The aims of our study were (1) to explore the differential expression of miRNAs in follicular fluid of women with PCOS compared with non-PCOS controls and (2) to investigate the role of the differential expressed miRNAs in pathological defects in PCOS, such as dysfunction of granulosa cells or abnormal folliculogenesis.

Based on miRNA-sequencing results, we found that the expression of miR-335-5p was significantly decreased in PCOS patients as compared with nonPCOS women, which suggested that miR-335-5p might be associated with PCOS. Subsequently, we studied the downstream targets of miR-335-5p and its possible mechanism in PCOS, which might provide a promising molecular target for PCOS treatment in the future.

\section{Materials and methods}

\section{Patients}

In this study, a total of 106 patients were recruited from the Center of Reproductive Medicine, Ren Ji Hospital, School of Medicine, Shanghai Jiao Tong University, between April 2015 and April 2016. The Ethics Committee of Ren Ji Hospital approved the study and all women gave their written informed consent before participation. Fifty-five of them were PCOS patients. The diagnosis of PCOS was performed according to the Rotterdam Consensus (2004). Fifty-one non-PCOS patients underwent IVF or intracytoplasmic sperm injection (ICSI) because of tubal factor or male factor infertility. In this study, participants with genetic diseases, thyroid diseases, a family history of diabetes and serious medical and surgical disease complications were excluded.

\section{Human follicular fluid collection}

Patients received human chorionic gonadotrophin during IVF/ ICSI when the diameter of the follicle was $>18 \mathrm{~mm}$. At the time of oocyte retrieval, the fluid from the first aspirated dominant follicle was collected. The fluid was centrifuged for $10 \mathrm{~min}$ at $2500 \mathrm{~g}$ to remove blood and debris. The supernatant was then collected and cryopreserved at $-80^{\circ} \mathrm{C}$.

\section{Library construction and miRNA sequencing}

The follicular fluids from three PCOS patients and three nonPCOS patients were used for miRNA sequencing. According to the manufacturer's instructions, Ion Total RNA-Seq Kit, v2.0 (Life Technologies) was used to construct cDNA libraries for single-end sequencing. PAGE was applied for size selection of the miRNA-sequencing cDNA library, which was then processed for the proton sequencing process. The mixture of samples that were diluted and mixed was processed on a OneTouch 2 instrument (Life Technologies) and enriched on a OneTouch 2 ES station (Life Technologies) for preparing the template-positive Ion PI Ion Sphere Particles (Life Technologies) according to Ion PI Hi-Q OT2 200 Kit (Life Technologies). After enrichment, according to Ion PI Hi-Q Sequencing 200 Kit (Life Technologies), the mixed samples were loaded on to 1 v3 Proton Chip (Life Technologies) and sequenced on Proton Sequencers. Furthermore, the EB-Seq algorithm was used to identify differential expressed miRNAs using the following criteria: false discovery rate $(\mathrm{FDR})<0.05$.

\section{Bioinformatics analysis}

Potential miRNA targets were identified in the miRanda database (http://www.microrna.org/). Gene ontology (GO) analysis was used to study biological processes for the target genes of differential expressed miRNAs. The GO annotations from NCBI (http://www.ncbi.nlm.nih.gov/), UniProt (http:// www.uniprot.org/) and the Gene Ontology (http://www. geneontology.org/) were downloaded. To identify the significant GO categories, Fisher's exact test was applied to calculate the $P$ values and the result of multiple hypothesis was used to correct the FDR (Zeeberg et al. 2003, Al-Shahrour et al. 2004). Kyoto Encyclopedia of Genes and Genomes (KEGG) pathway enrichment analysis was used to identify significant signaling pathways involving the differential expressed miRNA. Fisher's exact test was applied again to identify the significant pathways. In addition, we performed miRNA-mRNA network analysis according to the significant GO analysis and KEGG pathways.

\section{MicroRNA extraction and purification}

Additional dominant follicular fluid from 14 PCOS and 16 non-PCOS patients were used to validate the miRNAsequencing results. According to manufacturer's instructions, RNA from follicular fluid was extracted using the TRIzol LS Reagent (Invitrogen). Firstly, $0.25 \mathrm{~mL}$ of follicular fluid was added gently to $0.75 \mathrm{~mL}$ of TRIzol reagent. After incubating the mixture for $5 \mathrm{~min}, 0.2 \mathrm{~mL}$ of chloroform was added. The samples were then mixed vigorously for $15 \mathrm{~s}$ and incubated for another $10 \mathrm{~min}$ at room temperature. The samples were centrifuged at $12,000 \mathrm{~g}$ for $15 \mathrm{~min}$ at $4^{\circ} \mathrm{C}$, and the upper clarified phase was slowly transferred to a new tube. Afterward, $0.5 \mathrm{~mL}$ of $100 \%$ isopropanol was added. The samples were centrifuged at $12,000 \mathrm{~g}$ for $20 \mathrm{~min}$ at $4^{\circ} \mathrm{C}$ after incubating at $-20^{\circ} \mathrm{C}$ for $3 \mathrm{~h}$. The mixture was washed with $1 \mathrm{~mL}$ of $75 \%$ ethanol and centrifuged at $12,000 \mathrm{~g}$ for $5 \mathrm{~min}$ at $4^{\circ} \mathrm{C}$. The RNA quality was then determined using a NanoDrop ND-2000 spectrophotometer (Thermo Fisher Scientific) after adding $20 \mu \mathrm{L}$ of RNase-free water. Finally, $250 \mathrm{ng}$ of total RNA was reverse-transcribed to cDNA using an miRNeasy Mini Kit (Qiagen).

\section{Isolation of human granulosa cells}

After oocyte retrieval, human granulosa cells from 38 PCOS and 32 non-PCOS patients were used to verify target genes. The granulosa cells were collected and purified as described previously (Iwase et al. 2009). In brief, the pellets were purified by density centrifugation with Ficoll-Paque (GE-Health Care Bio-Science) and then digested in hyaluronidase (Sigma) at 
$37^{\circ} \mathrm{C}$ for $7 \mathrm{~min}$. The granulosa cells were also stored at $-80^{\circ} \mathrm{C}$ until RNA or protein extraction.

\section{Quantitative real-time PCR}

Total RNA from granulosa cells and the human granulosa-like tumor cell line, KGN, were extracted using an RNA isolation kit (Foregene), according to the manufacturer's instructions and quantified with NanoDrop ND-2000 spectrophotometer. RNA (300 ng) was reverse-transcribed into cDNA using the PrimeScript RT Master Mix Perfect Real-Time kit (TaKaRa). The miRNA and mRNA expression levels were then assessed using quantitative real-time polymerase chain reaction (qRT-PCR) with a SYBR RT-PCR kit (TaKaRa). The primer sequences for miRNAs and mRNAs were hsa-miR-335-5p, TCA AGA GCA ATA ACG AAA AAT GT; hsa-miR-27b-3p, TTC ACA GTG GCT AAG TTC TGC; hsa-miR-660-5p, TAC CCA TTG CAT ATC GGA GTT G; U6, forward: GGA ACG ATA CAG AGA AGA TTA GC, reverse: TGG AAC GCT TCA CGA ATT TGC G; SGK3, forward: AAA GAC GAG CAG GAC TAA ACG, reverse: GGA CTG TCC ATT TGA AGG AAT GC and ACTB, forward: GGA CTT CGA GCA AGA GAT GG, reverse: AGC ACT GTG TTG GCG TAC AG. The reactions were performed using the following program in an ABI PRISMH7700 sequence detector system (Life Technologies, Inc.): $5 \mathrm{~min}$ at $95^{\circ} \mathrm{C}$; followed by 40 cycles of $10 \mathrm{~s}$ at $95^{\circ} \mathrm{C}, 20 \mathrm{~s}$ at $59^{\circ} \mathrm{C}$ and $20 \mathrm{~s}$ at $72^{\circ} \mathrm{C}$; ending with a $5 \mathrm{~min}$ extension at $72^{\circ} \mathrm{C}$. Relative expression was normalized to the internal control (ACTB for mRNA or U6 small nuclear RNA for miRNA). Currently, there is no standard for an internal control for miRNAs. U6 RNA was used as a reference for miRNA analysis in follicular fluid in previous studies (Sang et al. 2013, Zhang et al. 2017a). The fold changes were analyzed using the $2^{\Delta \Delta C T}$ (cycle threshold) method (Livak \& Schmittgen 2001).

\section{Western blotting analysis}

Total protein was extracted using ice cold radioimmunoprecipitation assay (RIPA) lysis buffer (Cwbio), containing a phosphatase inhibitor (Roche) and a protease inhibitor cocktail (Roche). Then, $25 \mu \mathrm{g}$ of protein from each sample was electrophoresed in SDS polyacrylamide gels and then transferred to PVDF membranes. The membranes were blocked with $5 \%$ non-fat milk for $1 \mathrm{~h}$ at room temperature and then incubated with antibodies against SGK3 (serum/ glucocorticoid-regulated kinase family member 3; 1:500 dilution; Proteintech), mTOR (mammalian target of rapamycin kinase; 1:1000 dilution; Cell Signaling), Phospho-mTOR (1:1000 dilution; Ser2448, Cell Signaling), AKT (protein kinase B; 1:1000 dilution; Cell Signaling), Phospho-AKT (1:1000 dilution; Ser473, Cell Signaling) overnight at $4{ }^{\circ} \mathrm{C}$. After washing four times with Tris-buffered saline containing $0.1 \%$ Tween-20, the membranes were incubated with the appropriated secondary antibody, conjugated with horseradish peroxidase (Proteintech) for $1 \mathrm{~h}$ at room temperature. Bands with peroxidase activity were detected by an enhanced chemiluminescent detection system (Merck Millipore) and visualized using a G-Box chemiluminescence image capture system (Syngene). GAPDH (glyceraldehyde-3-phosphate dehydrogenase; 1:5000 dilution; Proteintech) as a protein loading control.

\section{Cell culture and transfection}

The KGN cell line is undifferentiated and possesses the physiological characteristics of ovarian cells. Thus, KGN cells were used to reveal the roles of miR-335-5p in this study. The cells were cultured in phenol red-free Dulbecco's modified Eagle's medium (DMEM)/F12 medium (Gibco) supplemented with $10 \%$ charcoal-stripped fetal bovine serum (FBS; Blood Bioind Stem Origin, Israel), $0.1 \mathrm{mg} / \mathrm{mL}$ streptomycin sulfate and $100 \mathrm{U} / \mathrm{mL}$ penicillin G (Invitrogen) in a humidified incubator at $37^{\circ} \mathrm{C}$ with $5 \% \mathrm{CO}_{2}$. Cells were seeded in sixwell dishes at $1 \times 10^{5}$ cells per well. The miR-335-5p mimics (sense: UCA AGA GCA AUA ACG AAA AAU GU, antisense: CAU UUU UCG UUA UUG CUC UUG A), corresponding scrambled control (sense: UUU GUA CUA CAC AAA AGU ACU G, antisense: CAG UAC UUU UGU GUA GUA CAA A), miR-335-5p inhibitor (sense: ACA UUU UUC GUU AUU GCU CUU GA) and corresponding scrambled control (sense: CAG UAC UUU UGU GUA GUA CAA A) were purchased from Ribobio (Guangzhou, China). According to the manufacturer's protocol, either miRNA mimics $(50 \mathrm{nM} /$ well) or inhibitor $(100 \mathrm{nM} /$ well $)$ and their corresponding scrambled controls were transfected into KGN cells using Lipofectamine 3000 (Invitrogen) $(7.5 \mu \mathrm{L} /$ well) diluted in $125 \mu \mathrm{L}$ of opti-MEM Medium (Gibco). The medium was replaced by fresh medium $24 \mathrm{~h}$ after transfection, and cells were cultured for another $24 \mathrm{~h}$.

\section{Dual-luciferase reporter assay}

The SGK3-3' UTR and the SGK3-mutated-3' UTR fragment were amplified by qRT-PCR. The fragment was then cloned into the Psicheck luciferase promoter vector (Promega) to develop the Luc-Psicheck-SGK3-3' UTR and Luc-PsicheckSGK3-mut-3' UTR vectors. KGN cells in 24-well plates were co-transfected with Luc-Psicheck-SGK3-3' UTR, LucPsicheck-SGK3-mut-3' UTR and miR-335-5p mimics or its scrambled control using Lipofectamine 3000 reagent (Invitrogen) according to the instructions of the manufacturer. In addition, cells were transfected with the pRL-SV40 vector as an internal standard. After transfection for $48 \mathrm{~h}$, Renilla and firefly luciferase activities were measured using the dualluciferase reporter assay system (Promega, Cat. No. E1910). The Renilla luciferase activity was normalized to the firefly luciferase activity.

\section{Cell counting Kit-8 assay}

Cell proliferation was assessed using the cell counting kit-8 assay (CCK-8) method. To assess the effect of miR-335-5p on proliferation, cells were transfected with miRNAs for $24 \mathrm{~h}$ and then reseeded in 96 -well plates at $5 \times 10^{3}$ cells $/ 100 \mu \mathrm{L} /$ well. The cells were cultured for 24,48 and $72 \mathrm{~h}$. According to the protocol of the manufacturer, CCK-8 solution was added to each well (MCE, USA). The absorbance was read at $450 \mathrm{~nm}$ 
after incubating for $3 \mathrm{~h}$. The assay was set in sextuplicate and repeated three times.

\section{Statistical analysis}

All the results were presented as mean \pm standard error of the mean (S.E.M.). All data were checked for their normal distribution by submission to the Kolmogrov-Smirnov test. After normal distribution test, we used the paired or unpaired Student's $t$-test to determine the significance of the observed differences. We applied Spearman rank correlations to compare and correlate quantitative variables. All statistical analyses were performed using SPSS 22 for Windows (IBM). $P<0.05$ was considered statistically significant.

\section{Results}

\section{Basic characteristics of the human subjects}

The clinical characteristics of all participants (55 PCOS and 51 non-PCOS) are summarized in Table 1. No significant differences were observed in terms of age, duration of infertility and levels of follicle-stimulating hormone $(\mathrm{FSH})$, basic estradiol (E2) and prolactin between the PCOS and non-PCOS groups. However, $\mathrm{BMI}$, and the level of total testosterone (TT), luteinizing hormone $(\mathrm{LH})$ and anti-Mullerian hormone $(\mathrm{AMH})$, the antral follicle count (AFC) and the $\mathrm{LH} / \mathrm{FSH}$ ratio were significantly increased in the patients with PCOS compared with those in the non-PCOS group $(P<0.05)$. Furthermore, the average number of retrieved oocytes in the patients with PCOS was higher than that in the nonPCOS group $(P<0.05)$.

\section{Differential miRNA expression in human follicular fluid samples}

Based on the deep miRNA-sequencing results, we obtained a total of 75,942,083 sequences reads, among

Table 1 Basic clinical characteristic of the participants.

\begin{tabular}{lrrr}
\hline & PCOS $(n=55)$ & NC $(n=51)$ & $P$ value \\
\hline Age (years) & $28.13 \pm 0.41$ & $27.37 \pm 0.46$ & 0.225 \\
Duration of infertility (year) & $3.47 \pm 0.23$ & $2.88 \pm 0.23$ & 0.075 \\
BMI $\left(\mathrm{kg} / \mathrm{m}^{2}\right)$ & $23.25 \pm 0.45$ & $21.62 \pm 0.38$ & 0.007 \\
FSH $(\mathrm{mlU} / \mathrm{mL})$ & $6.00 \pm 0.20$ & $6.23 \pm 0.16$ & 0.362 \\
$\mathrm{LH}(\mathrm{mIU} / \mathrm{mL})$ & $9.16 \pm 0.81$ & $4.56 \pm 0.28$ & $<0.001$ \\
$\mathrm{LH} / \mathrm{FSH}$ ratio & $1.51 \pm 0.11$ & $0.74 \pm 0.04$ & $<0.001$ \\
Basic E2 $(\mathrm{pg} / \mathrm{mL})$ & $37.06 \pm 2.46$ & $39.73 \pm 4.25$ & 0.581 \\
TT $(\mathrm{nmol} / \mathrm{L})$ & $1.33 \pm 0.09$ & $0.83 \pm 0.06$ & $<0.001$ \\
Prolactin $(\mathrm{ng} / \mathrm{mL})$ & $16.93 \pm 1.03$ & $19.66 \pm 1.40$ & 0.116 \\
AMH $(\mathrm{ng} / \mathrm{mL})$ & $12.31 \pm 0.77$ & $5.09 \pm 0.23$ & $<0.001$ \\
AFC $(n)$ & $22.78 \pm 1.02$ & $14.76 \pm 0.52$ & $<0.001$ \\
Retrieved oocytes $(n)$ & $18.58 \pm 0.99$ & $12.45 \pm 0.65$ & $<0.001$ \\
\hline
\end{tabular}

Values are presented as mean \pm S.E.M. Significant statistical difference $(P<0.05)$.

AFC, antral follicle count; $\mathrm{AMH}$, anti-Mullerian hormone; BMI, body mass index; E2, estradiol; FSH, follicle-stimulating hormone; $\mathrm{LH}$,

luteinizing hormone; NC, negative control; TT, total testosterone. which 16,523,445 sequences reads were derived from 2376 miRNAs, which were perfectly matched in the human miRNAs database (miRBase v21). Moreover, the EBSeq algorithm was used to identify miRNAs that were differentially expressed between the PCOS and nonPCOS group. Using our designated criteria of an FDR $<0.05$ and a fold change $>1.5$, a total of 19 differential expressed miRNAs were identified in the PCOS group compared with the non-PCOS group, including 3 upregulated and 16 downregulated miRNAs (Table 2), which were shown in the heatmap (Fig. 1A).

\section{The role of miRNAs in targeting essential genes}

GO analysis and KEGG pathway enrichment analysis were used to identify significantly enriched biological processes associated with the differential expressed miRNAs between PCOS and non-PCOS patients (shown in Fig. $1 \mathrm{~B}$ and $\mathrm{C}$ ). Many biological processes, including the FoxO signaling pathway, NF-kappa B signaling pathway, MAPKs signaling pathway, Wnt signaling pathway and PI3K-AKT signaling pathway were shown to be enriched. All these signaling pathways are correlated to cell proliferation, differentiation and apoptosis (Chang et al. 2017, Ma et al. 2017, Lu et al. 2018). These results suggest that the differential expressed miRNA in human follicular fluid were potentially related to cell proliferation and differentiation processes during folliculogenesis and oocyte maturation.

\section{Verification of miRNAs expression}

To validate the reliability of the miRNA-sequencing data, we detected the abundance of hsa-miR-335-5p, hsamiR-27b-3p, and hsa-miR-660-5p, which were overtly different between the PCOS and non-PCOS groups, using qRT-PCR. We chose miR-335-5p, miR-27b-3p and miR-660-5p due to their high abundance in follicular fluid. Furthermore, the heatmap figure showed these three miRNA samples were homogenous (Fig. 1A). The results showed that the abundance of miR-335-5p was significantly decreased in the follicular fluid from the patients with PCOS compared with that in the follicular fluid from the non-PCOS patients $(P<0.01)$, which was in consistent with the sequencing results (Fig. 2A). The levels of hsa-miR-27b-3p and hsa-miR-660-5p were decreased in the PCOS group; however, the differences were not significant (Fig. 2B and C).

\section{Correlation of basic clinical features with the expression of $\operatorname{miR}-335-5 p$}

Many clinical features were correlated with the abundance of miR-335-5p in follicular fluid. In our studied subjects, the abundance of miR-335-5p in human follicular fluid was significantly negatively correlated with AFC $(\rho=-0.623, P=0.001), \mathrm{AMH}$ $(\rho=-0.570, P=0.01)$ and TT $(\rho=-0.707, P=0.001)$. 
Table 2 Differentially expressed miRNAs of PCOS group compared with non-PCOS group (FDR $<0.05$ ).

\begin{tabular}{|c|c|c|c|c|c|}
\hline miRNA & $\operatorname{PCOS}(n=3)$ & $\mathbf{N C}(n=3)$ & $\log 2 \mathrm{FC}$ & FDR & Trend \\
\hline hsa-miR-664a-3p & 212.97 & 102.33 & 1.06 & 8.46759E-08 & Up \\
\hline hsa-miR-200c-3p & 2238.49 & 67.33 & 5.06 & 0.016756936 & Up \\
\hline hsa-miR-449b-5p & 97.53 & 24.30 & 2.01 & 0.036780595 & Up \\
\hline hsa-miR-202-5p & 36372.76 & 75295.85 & -1.05 & 0 & Down \\
\hline hsa-miR-335-5p & 1974.33 & 5536.41 & -1.49 & $2.4869 \mathrm{E}-14$ & Down \\
\hline hsa-miR-660-5p & 2329.12 & 3916.78 & -0.75 & $1.07248 \mathrm{E}-13$ & Down \\
\hline hsa-miR-185-5p & 3837.98 & 5791.81 & -0.60 & $2.81261 \mathrm{E}-08$ & Down \\
\hline hsa-miR-188-5p & 188.50 & 378.05 & -1.000 & $6.75422 \mathrm{E}-05$ & Down \\
\hline hsa-miR-507 & 42.85 & 128.51 & -1.580 & 0.000296398 & Down \\
\hline hsa-miR-513c-3p & 10.05 & 59.57 & -2.57 & 0.000308073 & Down \\
\hline hsa-miR-509-5p & 245.28 & 806.62 & -1.72 & 0.000317965 & Down \\
\hline hsa-miR-501-3p & 339.47 & 512.14 & -0.59 & 0.000679795 & Down \\
\hline hsa-miR-30a-3p & 1647.71 & 2749.27 & -0.74 & 0.001498431 & Down \\
\hline hsa-miR-513a-3p & 17.15 & 72.55 & -2.08 & 0.001823906 & Down \\
\hline hsa-miR-22-5p & 1031.50 & 2094.05 & -1.02 & 0.006488394 & Down \\
\hline hsa-miR-27b-3p & 7842.16 & 13488.83 & -0.78 & 0.009647922 & Down \\
\hline hsa-miR-5196-3p & 10.98 & 38.47 & -1.81 & 0.010461877 & Down \\
\hline hsa-miR-1224-5p & 63.68 & 129.00 & -1.02 & 0.021641476 & Down \\
\hline hsa-miR-548ak & 10.02 & 28.69 & -1.52 & 0.022263186 & Down \\
\hline
\end{tabular}

FDR, false discovery rate; NC, negative control; PCOS, polycystic ovarian syndrome.

However, the miR-335-5p level in human follicular fluid was not associated with BMI $(\rho=-0.256$, $P=0.172)$, the number of retrieved oocytes $(\rho=-0.323$, $P=0.082), \mathrm{FSH}$ and $\mathrm{LH}(\rho=0.022, P=0.911 ; \rho=0.202$, $P=0.283$, respectively). These results indicate that a low abundance of miR-335-5p might be correlated with abnormal folliculogenesis in PCOS.

\section{Network analysis of miR-335-5p}

The results of miR-335-5p-mRNA network were shown in Fig. 3. KEGG pathway enrichment analysis revealed important roles of miR-335-5p in the folliculogenesis process and the normal function of ovaries, including the Wnt, MAPK, TGFB and PI3K-AKT signaling pathways. GO analysis demonstrated certain biological functions associated with miR-335-5p, such as embryo development, antral ovarian follicle growth and regulation of cell proliferation. This figure also showed certain potential target genes of miR-335-5p, such as SGK3, FOXO3, TGFBR1 and CD47. Up to now, little is known about the role of $S G K 3$ in granulosa cells. Thus, SGK3 was selected as target gene in our study.

\section{miR-335-5p directly inhibits SGK3 expression}

To predict the target genes of miR-335-5p, we used bioinformatics prediction software to search for its direct mRNA targets. According to KEGG and GO analysis results, SGK3 caught our attention among the predicted target genes of miR-335-5p. To determine whether SGK3 is a direct target gene of miR-335-5p, we cloned the SGK3 wild-type 3' UTR (wt 3' UTR) or a deletion mutated sequence (mut $3^{\prime}$ UTR) into a reporter plasmid, downstream of the luciferase gene. The dualluciferase plasmid construction model was shown in Fig. 4A. The results showed that miR-335-5p mimics reduced the fluorescence of wt 3' UTR compared with
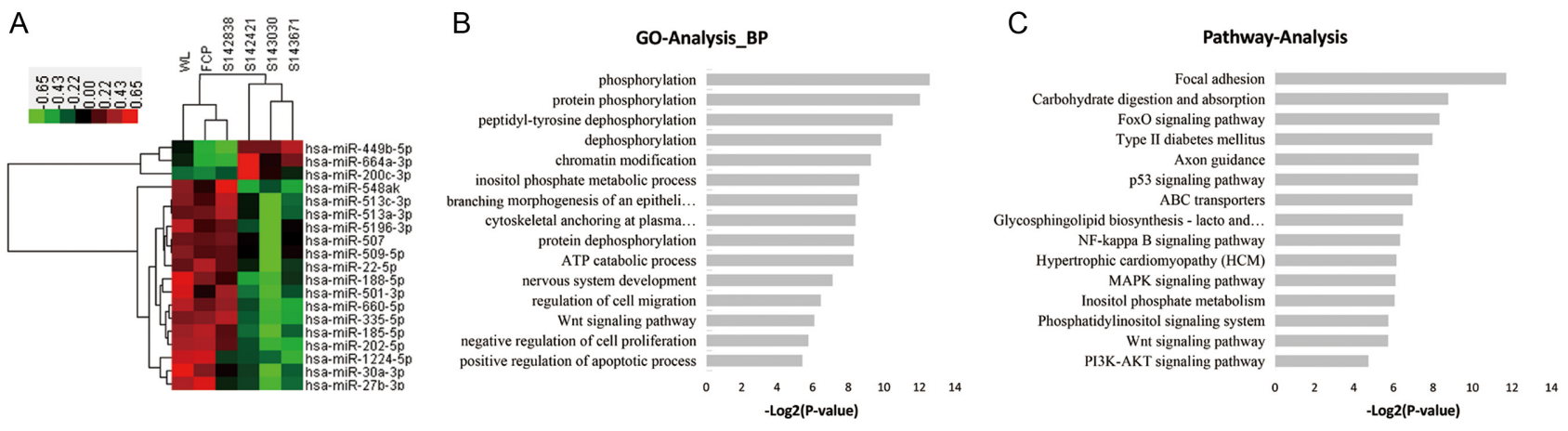

Figure 1 (A) Heatmap of the 19 miRNAs that were differentially expressed in the follicular fluid between PCOS ( $n=3)$ and non-PCOS groups $(n=3)$. The left three columns are the non-PCOS group and the right three columns are the PCOS group. Green to red equates to an increase in miRNA expression. (B) Gene ontology (GO) analysis of the biological processes of the target genes of the differential expressed miRNAs in follicular fluid. (C) KEGG pathway analysis of the target genes of the differential expressed miRNAs in the follicular fluid. 

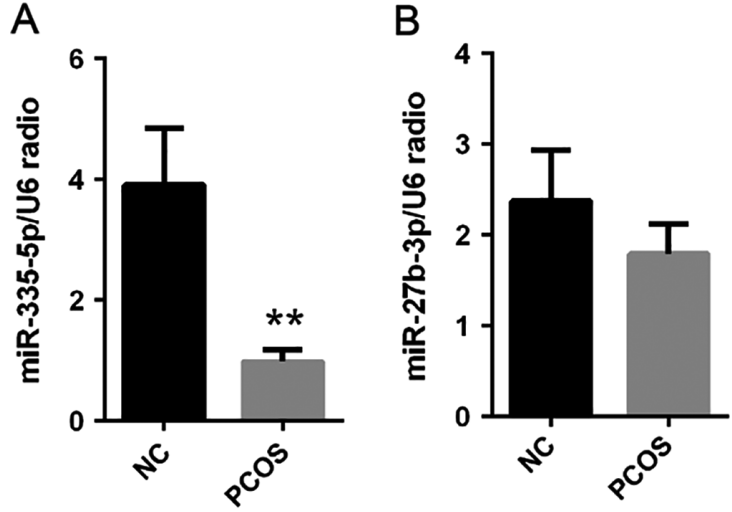

the control group (Fig. 4B, lanes 1, 2, 3 and 4) while had no effect on the fluorescence of mut 3' UTR (Fig. 4B, lanes 5 and 6). Furthermore, the mimics or inhibitor of miR-335-5p were transfected into KGN cells to observe the mRNA and protein expression levels of SGK3. The results showed that miR-335-5p mimics reduced, while miR-335-5p inhibitor increased, the mRNA and protein expression levels of SGK3 in KGN cells. These results suggest that $S G K 3$ is a direct target gene of miR-335-5p.

\section{miR-335-5p inhibited KGN cells proliferation}

Since SGK3 was a target gene regulated by miR-335-5p, and it was shown to affect the cell proliferation in many types of cells (Sun et al. 2016, Chen et al. 2018), we hypothesized that miR-335-5p might be associated with the growth and proliferation in granulosa cells. CCK-8

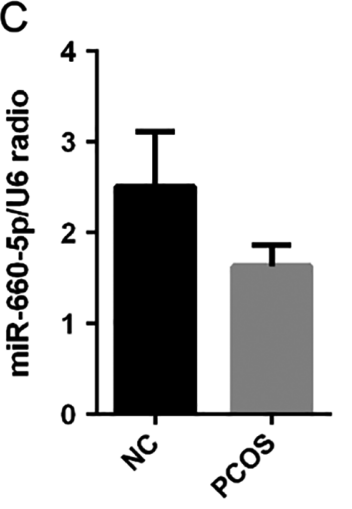

Figure 2 Verification of the miRNA-sequencing results by qRT-PCR in human follicular fluid. Differential abundance of miR-335-5p, miR-27b-3p and miR-660-5p by qRT-PCR in additional follicular fluid samples from PCOS $(n=14)$ and non-PCOS $(n=16)$ patients are shown in $(A),(B)$ and $(C)$, respectively. Significant statistical difference $(P<0.05)$. Student's $t$-test was used and all data are reported as mean \pm S.E.M. $* * P<0.01$. assay revealed that the miR-335-5p mimics significantly decreased cell proliferation, whereas the miR-335-5p inhibitor promoted the proliferation of KGN cells (Fig. 5A and B), suggesting that miR-335-5p may be a cell proliferation suppressor in granulosa cells.

\section{Effects of miR-335-5p on the PI3K-AKT signaling pathway}

In order to identify the signaling pathway that was involved in the regulation of cell proliferation by miR$335-5 p$, we measured the total protein levels of AKT and $\mathrm{mTOR}$, as well as the phosphorylation of AKT and mTOR, which was screened by KEGG pathway enrichment analysis (Fig. 1C) and was shown to affect the cell proliferation (Liu et al. 2018, Wang et al. 2018). The results showed that miR-335-5p mimics obviously

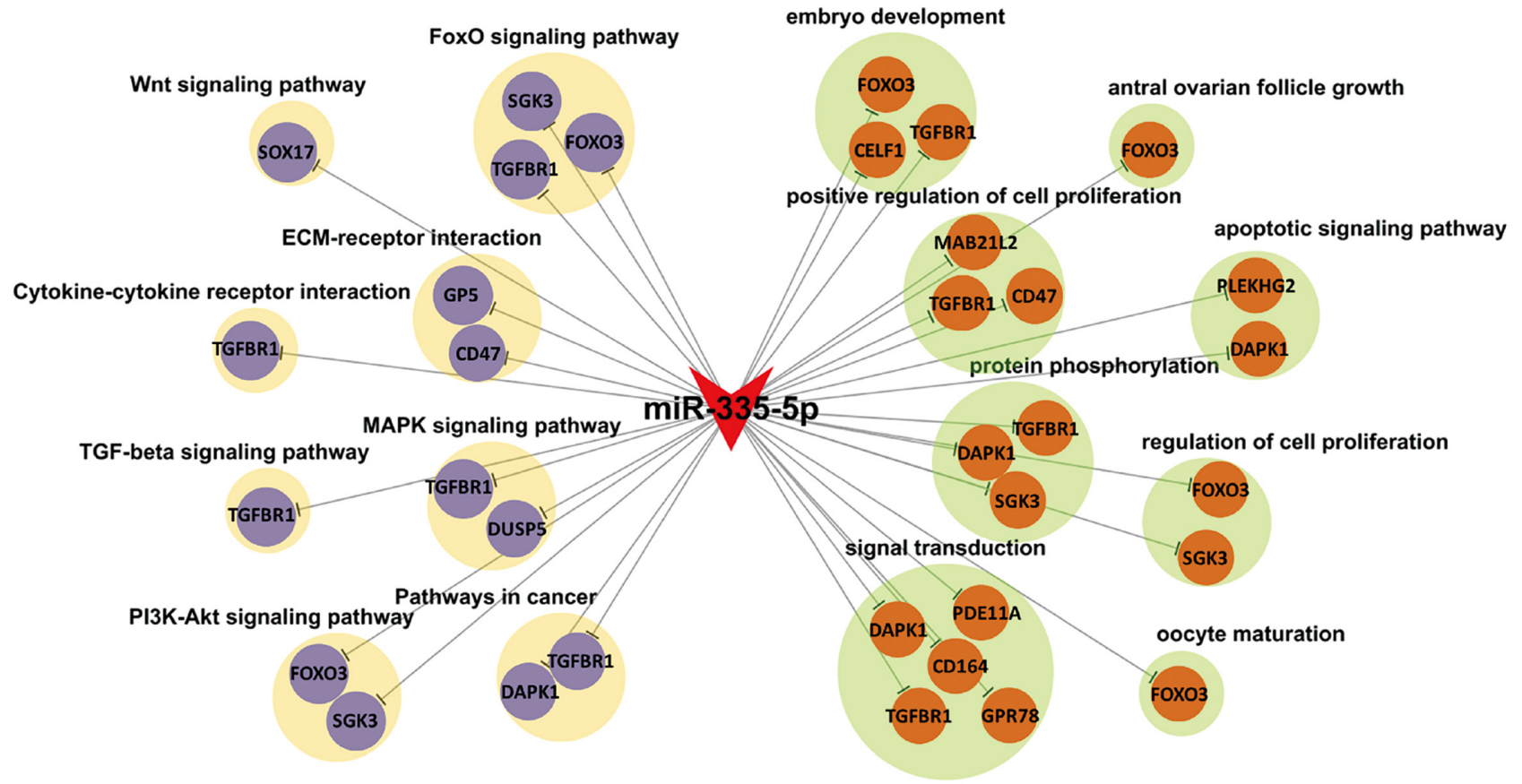

Figure 3 miRNA-mRNA network analysis of miR-335-5p according to the significant GO and KEGG pathways. The purple cycle nodes on the left represent genes identified by KEGG pathway analysis, and the orange cycle nodes represent genes identified by GO analysis. 
A

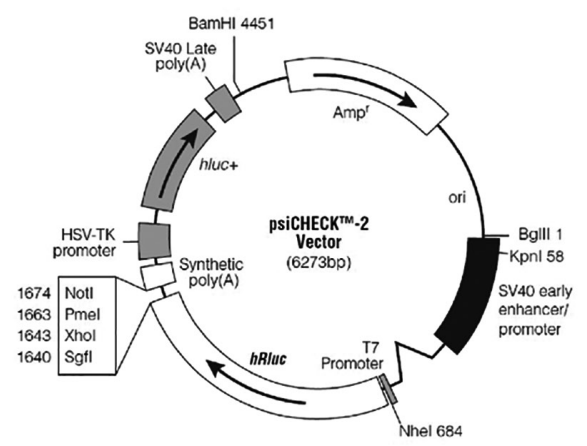

C

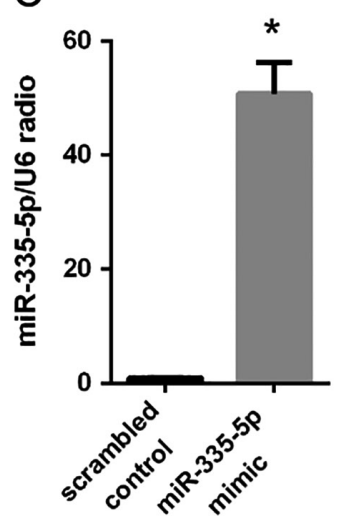

$\mathrm{F}$

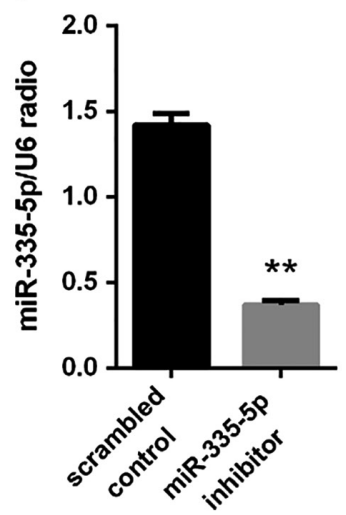

B

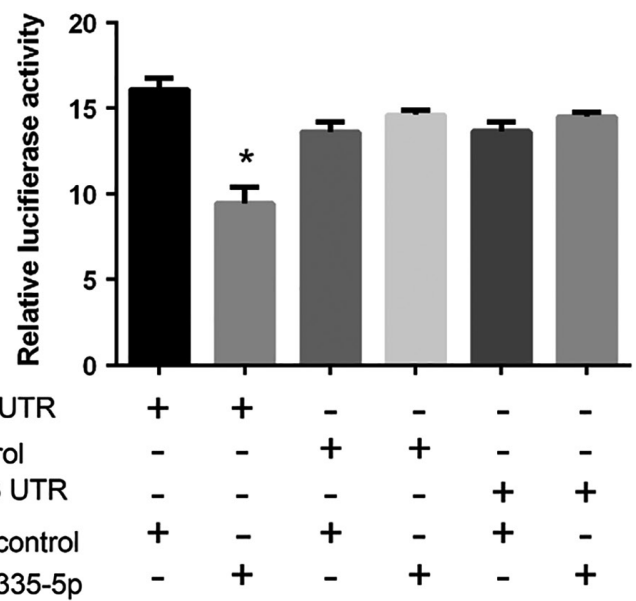

E

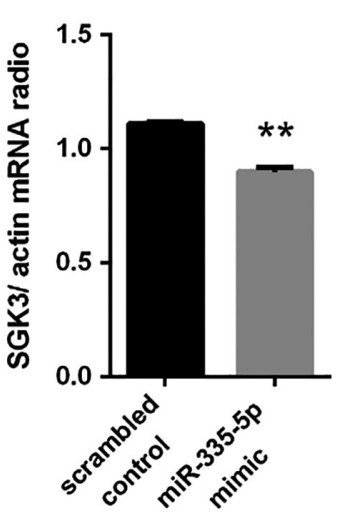

G

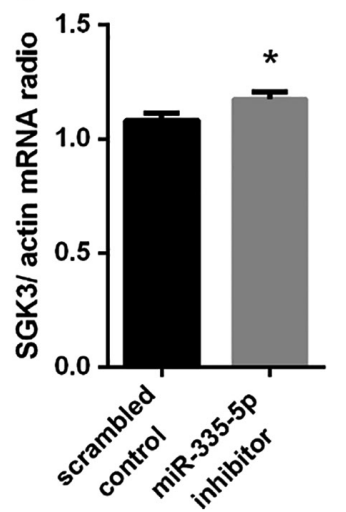

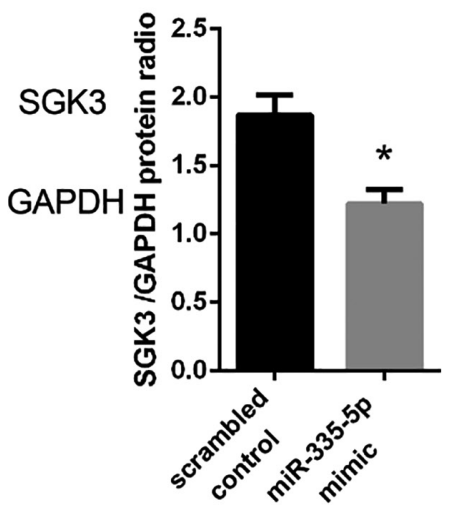

$\mathrm{H}$

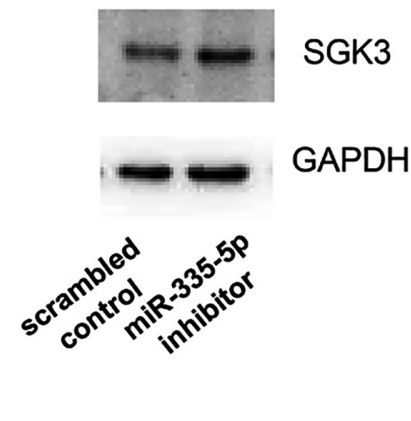

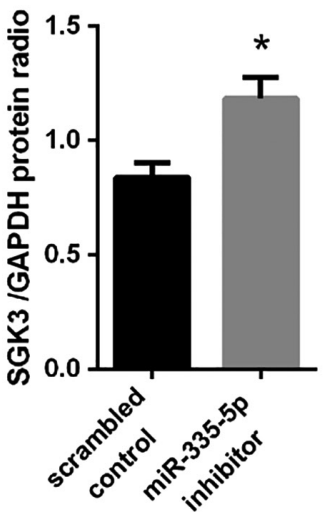

Figure 4 miR-335-5p directly inhibited SGK3 expression by binding to its 3'-untranslated region (UTR). (A) The dual-luciferase plasmid model of SGK3. (B) Validation of miR-335-5p binding to the SGK3 3'-UTR using the dual-luciferase reporter assay. (C) and (F) miR-335-5p mimics or inhibitor transfection efficiencies. (D) and (E) mRNA and protein levels of SGK3 after miR-335-5p mimics transfection. (G) and (H) mRNA and protein levels of SGK3 after miR-335-5p inhibitor transfection. The relative protein level was normalized to that of glyceraldehyde 3-phosphate dehydrogenase $(\mathrm{GAPDH})$. Significant statistical difference $(P<0.05)$. Student's $t$-test was used and all data are reported as mean \pm S.E.M. $* P<0.05$; $* * P<0.01$.

inhibited the activation of AKT and mTOR (Fig. 5C), as shown by the reduced the phosphorylation of AKT and mTOR. In contrast, the miR-335-5p inhibitor promoted the activation of AKT and mTOR. These results indicate that miR-335-5p might exert its effect through the PI3KAKT-mTOR signaling pathway. 

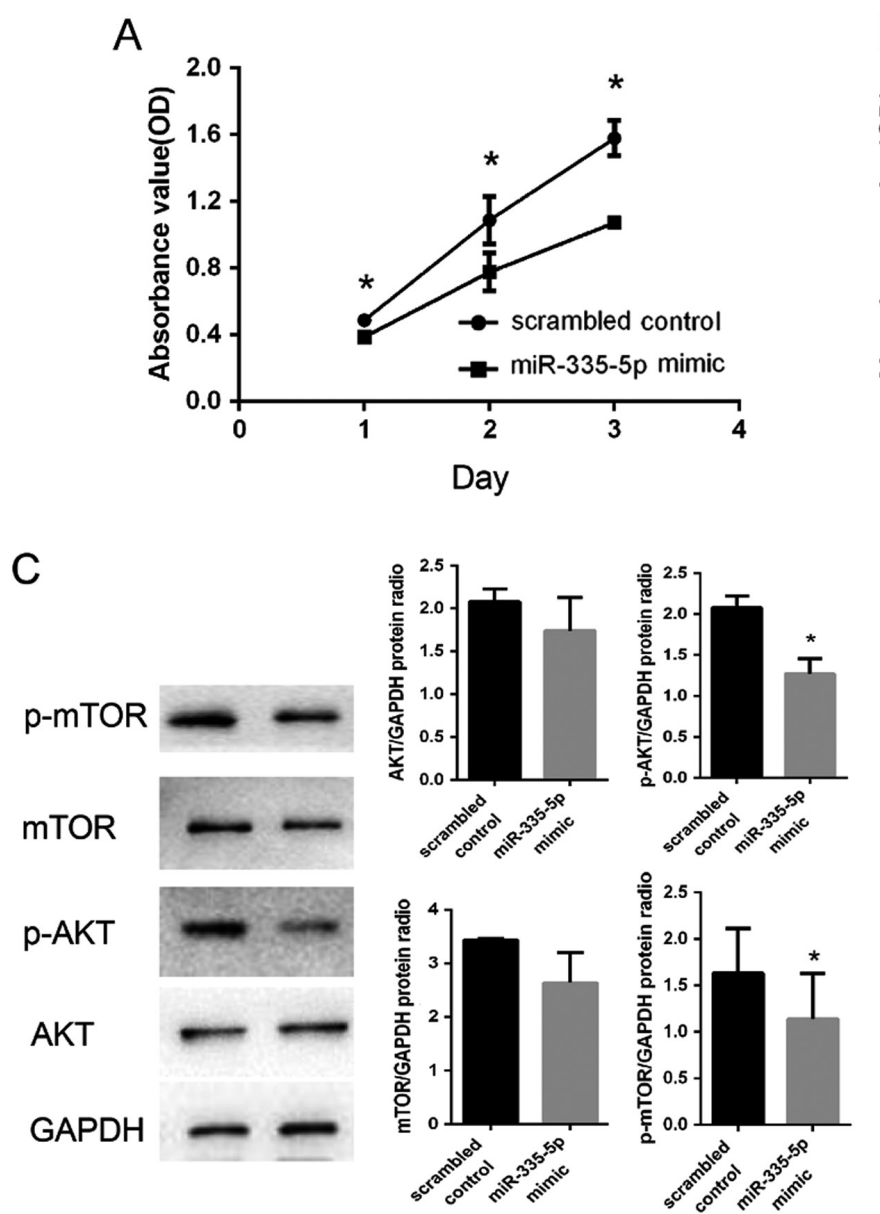
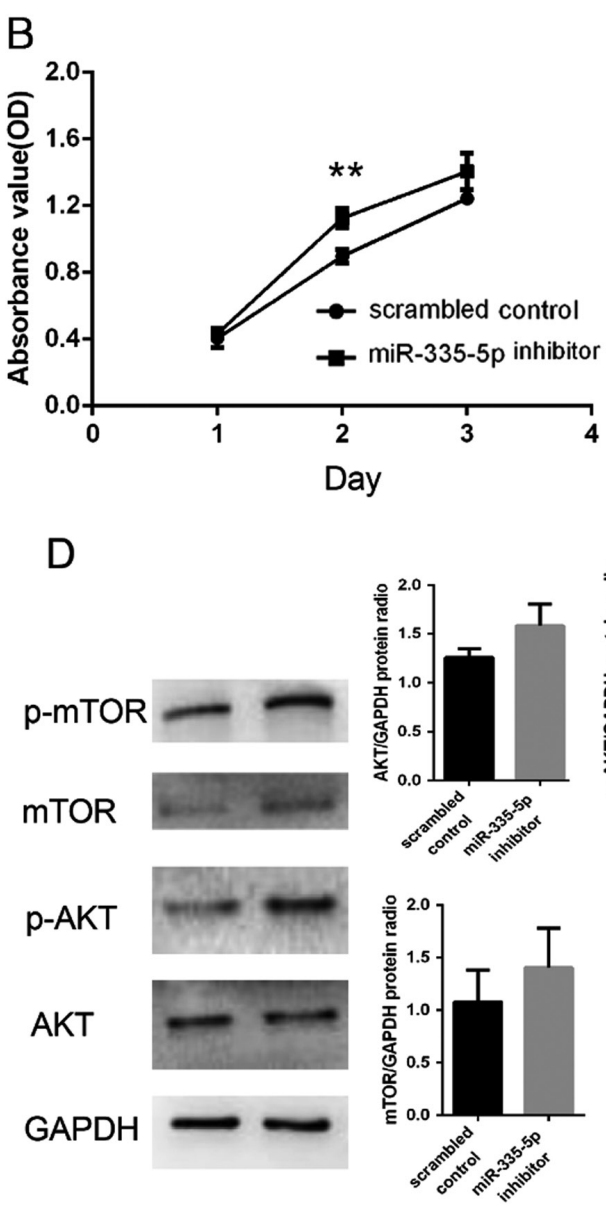
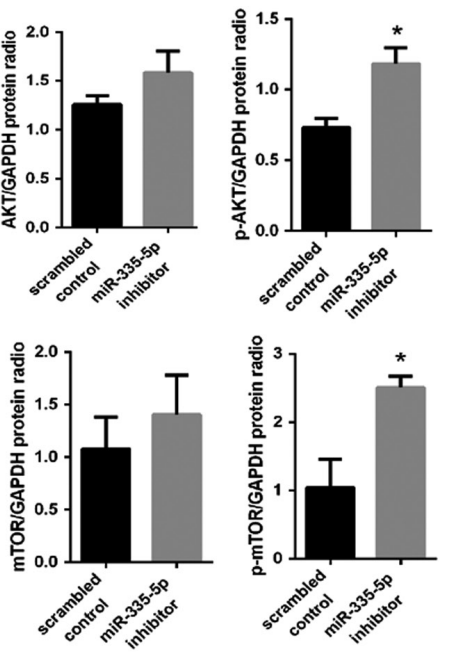

Figure 5 The effect of overexpression of miR-335-5p on the proliferation of KGN cells. (A) CCK-8 assay revealing that miR-335-5p mimics led to the suppression in proliferation. (B) The miR-335-5p inhibitor promoted the proliferation of KGN cells. (C) and (D) The protein expression levels of AKT, p-AKT, mTOR and p-mTOR in KGN cells after miR-335-5p mimics or inhibitor transfection. The relative protein level was normalized to that of glyceraldehyde 3-phosphate dehydrogenase (GAPDH). Significant statistical difference $(P<0.05)$. Student's $t$-test was used and all data are reported as mean \pm S.E.M. $* P<0.05, * * P<0.01$.

\section{SGK3 expression increased in PCOS granulosa cells}

In order to verify the association of SGK3 and miR335-5p with PCOS development, we further tested the abundance of miR-335-5p and SGK3 in human granulosa cells obtained from PCOS and non-PCOS patients. The results show that the abundance of miR$335-5 p$ was decreased in human granulosa cells from the PCOS group (Fig. 6A), which was consistent with the results in follicular fluid, while the abundance of SGK3 mRNA and protein were significantly increased in the PCOS group (Fig. 6B and C). These observations may indicate a crucial role of miR-335-5p and SGK3 in the development of PCOS.

\section{Discussion}

In this study, we used high-throughput sequencing to identify differential expressed miRNAs in human follicular fluid of PCOS and non-PCOS patients.
We observed a low abundance of miR-335-5p in the follicular fluid of patients with PCOS and explored its possible role in the pathogenesis of PCOS.

Our sequencing results demonstrated that 19 miRNAs were differentially expressed between the PCOS and non-PCOS groups. Among these differential expressed miRNAs, miR-335-5p was further verified to present a lower level in the follicular fluid of PCOS patients compared with that in non-PCOS patients by qRT-PCR. Furthermore, correlation analysis of miR-335-5p and clinical characteristics showed that miR-335-5p was negatively correlated with $\mathrm{AFC}$ and $\mathrm{AMH}$, which were proposed as predictors of ovarian reserve and clinical outcome in different infertility states (Broekmans et al. 2006). AMH prevents premature depletion of ovarian follicles and regulates steroidogenesis in granulosa cells (La Marca et al. 2009), and plasma AMH levels and AFC were significantly higher in patients with PCOS than in non-PCOS patients. Therefore, we believed that the abundance of miR-335-5p was related to 
A

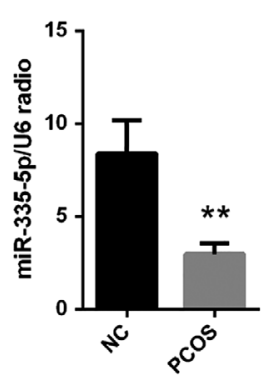

C

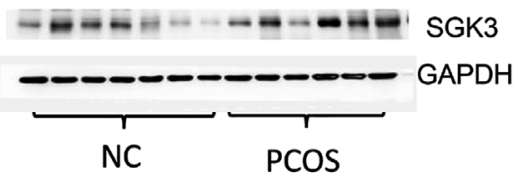

B
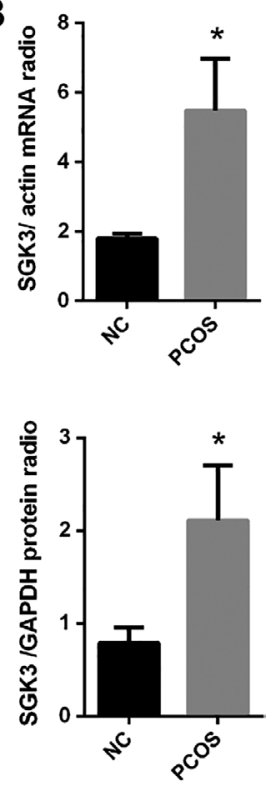

Figure 6 (A) and (B) The abundance of miR-335-5p and the mRNA level of SGK3 in the PCOS $(n=32)$ and non-PCOS $(n=25)$ groups. (C) The SGK3 protein level in the PCOS $(n=6)$ and non-PCOS $(n=7)$ group. The relative protein level was normalized to the level of glyceraldehyde 3-phosphate dehydrogenase (GAPDH). Significant statistical difference $(P<0.05)$. Student's $t$-test was used and all data are reported as the mean \pm S.E.M. $* P<0.05,{ }^{* *} P<0.01$.

endocrine abnormality and an increased number of growing follicles.

miR-335 was reported to be an anticancer agent in various organs (Scarola et al. 2010, Tome et al. 2011, Cao et al. 2013, Zhang et al. 2017b) and participate in the process of bone formation and differentiation (Zhang et al. 2011, Wang et al. 2017), which suggest that miR-335-5p may play an important role in cell proliferation and migration. However, there has been no report implying an effect of miR-335-5p in human follicular fluid and granulosa cells. Using GO and KEGG pathway analysis, as well as bioinformatics software, we revealed that $S G K 3$ was a potential target gene for miR$335-5 p$, which were confirmed by the dual-luciferase reporter assay and transfection of miR-335-5p mimics and inhibitors into KGN cells. KGN cell line was a valid model for granulosa cells. One study reported the developmental stage of KGN cells may be close to that of immature granulosa cells (Nishi et al. 2001).

The SGK family, including SGK1, SGK2 and SGK3, dominates a wide range of biological responses, including control of cell growth, proliferation, metabolism and intracellular material exchange possesses (Pearce et al. 2010). Alliston et al. demonstrated that SGK is located in the nucleus and regulates the proliferation and differentiation of granulosa cells (Alliston et al. 2000). Given the SGK3 is the direct inhibited by miR-335-5p and miR-335-5p was involved in the proliferation of

granulosa cells, we believed that the effect of miR$335-5 p$ on granulosa cells proliferation might be mediated by downregulation of SGK3 expression, which is further supposed by the findings that the abundance of SGK3 is higher, whereas the abundance of miR-335-5p is lower in granulosa cells in PCOS patients compared with those in non-PCOS subjects. Furthermore, GO and KEGG pathway analysis results also revealed that miR-335-5p is involved in PI3K-AKT-mTOR signaling pathway, a critical regulator of proliferation and differentiation of granulosa cells (Makker et al. 2014), which further confirmed by western blotting. Since SGK3 could activate mTORC1 by phosphorylating TSC complex subunit 2 (TSC2) (Bago et al. 2016), we suspected that the effect of miR-335-5p on granulosa cells proliferation might be mediated by activation of mTOR pathway through induction of SGK3 expression.

In conclusion, we confirmed that human follicular fluid contained large amounts of miRNAs, some of which were differentially expressed between non-PCOS and PCOS patients. Among these differential expressed miRNAs, miR-335-5p abundance was observed lower both in follicular fluid and granulosa cells obtained from PCOS patients as compared with non-PCOS patients. We further revealed that miR-335-5p suppressed granulosa cell proliferation, which might be mediated by mTOR pathway via targeting and inhibiting SGK3 expression. These observations provided a new perspective on the dysfunction of granulosa cells in PCOS and indicated that miR-335-5p could be a new molecular target to improve dysfunctional granulosa cells in PCOS.

\section{Declaration of interest}

The authors declare that there is no conflict of interest that could be perceived as prejudicing the impartiality of the research reported.

\section{Funding}

This study was supported by the National Natural Science Foundation of China under Grant (No. 81300546), Shanghai Natural Science Foundation (No. 13ZR1424600) and the Shanghai Key Laboratory for Assisted Reproduction and Reproductive Genetics under Grant (No. 17DZ2271100).

\section{Acknowledgments}

The authors gratefully acknowledge all study participants for their contributions.

\section{References}

Alliston TN, Gonzalez-Robayna IJ, Buse P, Firestone GL \& Richards JS 2000 Expression and localization of serum/glucocorticoid-induced kinase in the rat ovary: relation to follicular growth and differentiation. Endocrinology 141 385-395. (https://doi.org/10.1210/endo.141.1.7257) 
Al-Shahrour F, Diaz-Uriarte R \& Dopazo J 2004 FatiGO: a web tool for finding significant associations of Gene Ontology terms with groups of genes. Bioinformatics 20 578-580. (https://doi.org/10.1093/ bioinformatics/btg455)

Bago R, Sommer E, Castel P, Crafter C, Bailey FP, Shpiro N, Baselga J, Cross D, Eyers PA \& Alessi DR 2016 The hVps34-SGK3 pathway alleviates sustained PI3K/Akt inhibition by stimulating mTORC1 and tumour growth. EMBO Journa/ 35 1902-1922. (https://doi.org/10.15252/ embj.201693929)

Bartel DP 2009 MicroRNAs: target recognition and regulatory functions. Cell 136 215-233. (https://doi.org/10.1016/j.cell.2009.01.002)

Broekmans FJ, Kwee J, Hendriks DJ, Mol BW \& Lambalk CB 2006 A systematic review of tests predicting ovarian reserve and IVF outcome. Human Reproduction Update 12 685-718. (https://doi.org/10.1093/ humupd/dml034)

Cao J, Cai J, Huang D, Han Q, Yang Q, Li T, Ding H \& Wang Z 2013 miR-335 represents an invasion suppressor gene in ovarian cancer by targeting Bcl-w. Oncology Reports 30 701-706. (https://doi.org/10.3892/ or.2013.2482)

Carletti MZ \& Christenson LK 2009 MicroRNA in the ovary and female reproductive tract. Journal of Animal Science 87 E29-E38. (https://doi. org/10.2527/jas.2008-1331)

Chang DL, Wei W, Yu ZP \& Qin CK 2017 miR-152-5p inhibits proliferation and induces apoptosis of liver cancer cells by up-regulating FOXO expression. Die Pharmazie 72 338-343. (https://doi.org/10.1691/ ph.2017.7406)

Chen Y, Sun Z, Qi M, Wang X, Zhang W, Chen C, Liu J \& Zhao W 2018 INPP4B restrains cell proliferation and metastasis via regulation of the PI3K/AKT/SGK pathway. Journal of Cellular and Molecular Medicine 22 2935-2943. (https://doi.org/10.1111/jcmm.13595)

Das M, Djahanbakhch O, Hacihanefioglu B, Saridogan E, Ikram M, Ghali L, Raveendran M \& Storey A 2008 Granulosa cell survival and proliferation are altered in polycystic ovary syndrome. Journal of Clinical Endocrinology and Metabolism 93 881-887. (https://doi.org/10.1210/ jc.2007-1650)

Franks S, Stark J \& Hardy K 2008 Follicle dynamics and anovulation in polycystic ovary syndrome. Human Reproduction Update 14 367-378. (https://doi.org/10.1093/humupd/dmn015)

Ganepola GA, Rutledge JR, Suman P, Yiengpruksawan A \& Chang DH 2014 Novel blood-based microRNA biomarker panel for early diagnosis of pancreatic cancer. World Journal of Gastrointestinal Oncology 6 22-33. (https://doi.org/10.4251/wjgo.v6.i1.22)

Goodarzi MO, Dumesic DA, Chazenbalk G \& Azziz R 2011 Polycystic ovary syndrome: etiology, pathogenesis and diagnosis. Nature Reviews Endocrinology 7 219-231. (https://doi.org/10.1038/ nrendo.2010.217)

Hanrieder J, Nyakas A, Naessen T \& Bergquist J 2008 Proteomic analysis of human follicular fluid using an alternative bottom-up approach. Journal of Proteome Research 7 443-449. (https://doi.org/10.1021/pr070277z)

Hanrieder J, Zuberovic A \& Bergquist J 2009 Surface modified capillary electrophoresis combined with in solution isoelectric focusing and MALDI-TOF/TOF MS: a gel-free multidimensional electrophoresis approach for proteomic profiling - exemplified on human follicular fluid. Journal of Chromatography: Part A 1216 3621-3628. (https://doi. org/10.1016/j.chroma.2008.12.026)

Huan L, Bao C, Chen D, Li Y, Lian J, Ding J, Huang S, Liang L \& He X 2016 MicroRNA-127-5p targets the biliverdin reductase $\mathrm{B} /$ nuclear factorkappaB pathway to suppress cell growth in hepatocellular carcinoma cells. Cancer Science 107 258-266. (https://doi.org/10.1111/cas.12869)

Iwase A, Goto M, Harata T, Takigawa S, Nakahara T, Suzuki K, Manabe S \& Kikkawa F 2009 Insulin attenuates the insulin-like growth factor-I (IGF-I)-Akt pathway, not IGF-I-extracellularly regulated kinase pathway, in luteinized granulosa cells with an increase in PTEN. Journal of Clinical Endocrinology and Metabolism 94 2184-2191. (https://doi.org/10.1210/ jc.2008-1948)

Johnnidis JB, Harris MH, Wheeler RT, Stehling-Sun S, Lam MH, Kirak O, Brummelkamp TR, Fleming MD \& Camargo FD 2008 Regulation of progenitor cell proliferation and granulocyte function by microRNA-223. Nature 451 1125-1129. (https://doi.org/10.1038/nature06607)

La Marca A, Broekmans FJ, Volpe A, Fauser BC, Macklon NS \& ESHRE Special Interest Group for Reproductive Endocrinology - AMH Round Table 2009 Anti-Mullerian hormone $(\mathrm{AMH})$ : what do we still need to know? Human Reproduction 24 2264-2275. (https://doi.org/10.1093/ humrep/dep210)

Liu JY, Zeng QH, Cao PG, Xie D, Yang F, He LY, Dai YB, Li JJ, Liu XM, Zeng HL et al. 2018 SPAG5 promotes proliferation and suppresses apoptosis in bladder urothelial carcinoma by upregulating Wnt3 via activating the AKT/mTOR pathway and predicts poorer survival. Oncogene 37 3937-3952. (https://doi.org/10.1038/s41388-018-0223-2)

Livak KJ \& Schmittgen TD 2001 Analysis of relative gene expression data using real-time quantitative PCR and the 2(-Delta Delta C(T)) method. Methods 25 402-408. (https://doi.org/10.1006/meth.2001.1262)

Lu HJ, Jin PY, Tang Y, Fan SH, Zhang ZF, Wang F, Wu DM, Lu J \& Zheng YL 2018 microRNA-136 inhibits proliferation and promotes apoptosis and radiosensitivity of cervical carcinoma through the NF-kappaB pathway by targeting E2F1. Life Sciences 199 167-178. (https://doi.org/10.1016/j. Ifs.2018.02.016)

Ma Y, Fu S, Lu L \& Wang X 2017 Role of androgen receptor on cyclic mechanical stretch-regulated proliferation of $\mathrm{C} 2 \mathrm{C} 12$ myoblasts and its upstream signals: IGF-1-mediated PI3K/Akt and MAPKs pathways. Molecular and Cellular Endocrinology 450 83-93. (https://doi. org/10.1016/j.mce.2017.04.021)

Makker A, Goel MM \& Mahdi AA 2014 PI3K/PTEN/Akt and TSC/mTOR signaling pathways, ovarian dysfunction, and infertility: an update. Journal of Molecular Endocrinology 53 R103-R118. (https://doi. org/10.1530/JME-14-0220)

Moran L \& Teede H 2009 Metabolic features of the reproductive phenotypes of polycystic ovary syndrome. Human Reproduction Update $\mathbf{1 5}$ 477-488. (https://doi.org/10.1093/humupd/dmp008)

Nishi Y, Yanase T, Mu Y, Oba K, Ichino I, Saito M, Nomura M, Mukasa C, Okabe T, Goto K et al. 2001 Establishment and characterization of a steroidogenic human granulosa-like tumor cell line, $\mathrm{KGN}$, that expresses functional follicle-stimulating hormone receptor. Endocrinology 142 437-445. (https://doi.org/10.1210/endo.142.1.7862)

Patel SS \& Carr BR 2008 Oocyte quality in adult polycystic ovary syndrome. Seminars in Reproductive Medicine 26 196-203. (https://doi. org/10.1055/s-2008-1042958)

Pearce LR, Komander D \& Alessi DR 2010 The nuts and bolts of AGC protein kinases. Nature Reviews: Molecular Cell Biology 11 9-22. (https://doi.org/10.1038/nrm2822)

Revelli A, Delle Piane L, Casano S, Molinari E, Massobrio M \& Rinaudo P 2009 Follicular fluid content and oocyte quality: from single biochemical markers to metabolomics. Reproductive Biology and Endocrinology 7 40. (https://doi.org/10.1186/1477-7827-7-40)

Rodgers RJ \& Irving-Rodgers HF 2010 Formation of the ovarian follicular antrum and follicular fluid. Biology of Reproduction 82 1021-1029. (https://doi.org/10.1095/biolreprod.109.082941)

Rotterdam ESHRE/ASRM-Sponsored PCOS Consensus Workshop Group 2004 Revised 2003 consensus on diagnostic criteria and long-term health risks related to polycystic ovary syndrome (PCOS). Human Reproduction 19 41-47. (https://doi.org/10.1093/humrep/deh098)

Sang Q, Yao Z, Wang H, Feng R, Wang H, Zhao X, Xing Q, Jin L, He L, Wu L et al. 2013 Identification of microRNAs in human follicular fluid: characterization of microRNAs that govern steroidogenesis in vitro and are associated with polycystic ovary syndrome in vivo. Journal of Clinical Endocrinology and Metabolism 98 3068-3079. (https://doi.org/10.1210/ jc.2013-1715)

Scalici E, Traver S, Mullet T, Molinari N, Ferrieres A, Brunet C, Belloc S \& Hamamah S 2016 Circulating microRNAs in follicular fluid, powerful tools to explore in vitro fertilization process. Scientific Reports $\mathbf{6} 24976$. (https://doi.org/10.1038/srep24976)

Scarola M, Schoeftner S, Schneider C \& Benetti R 2010 miR-335 directly targets $\mathrm{Rb} 1$ (pRb/p105) in a proximal connection to p53dependent stress response. Cancer Research 70 6925-6933. (https://doi. org/10.1158/0008-5472.CAN-10-0141)

Shukla GC, Singh J \& Barik S 2011 MicroRNAs: processing, maturation, target recognition and regulatory functions. Molecular and Cellular Pharmacology 3 83-92.

Sun X, Liu X, Liu BO, Li S, Zhang D \& Guo H 2016 Serum- and glucocorticoid-regulated protein kinase 3 overexpression promotes tumor development and aggression in breast cancer cells. Oncology Letters 12 437-444. (https://doi.org/10.3892/ol.2016.4638)

Tome M, Lopez-Romero P, Albo C, Sepulveda JC, Fernandez-Gutierrez B, Dopazo A, Bernad A \& Gonzalez MA 2011 miR-335 orchestrates cell 
proliferation, migration and differentiation in human mesenchymal stem cells. Cell Death and Differentiation 18 985-995. (https://doi. org/10.1038/cdd.2010.167)

Wang Y, Yang T, Zhang Z, Lu M, Zhao W, Zeng X \& Zhang W 2017 Long non-coding RNA TUG1 promotes migration and invasion by acting as a ceRNA of miR-335-5p in osteosarcoma cells. Cancer Science $\mathbf{1 0 8}$ 859-867. (https://doi.org/10.1111/cas.13201)

Wang F, Tan WH, Liu W, Jin YX, Dong DD, Zhao XJ \& Liu Q 2018 Effects of miR-214 on cervical cancer cell proliferation, apoptosis and invasion via modulating PI3K/AKT/mTOR signal pathway. European Review for Medical and Pharmacological Sciences 22 1891-1898. (https://doi. org/10.26355/eurrev_201804_14711)

Ying SY, Chang DC, Miller JD \& Lin SL 2006 The microRNA: overview of the RNA gene that modulates gene functions. Methods in Molecular Biology 342 1-18. (https://doi.org/10.1385/1-59745-123-1:1)

Zeeberg BR, Feng W, Wang G, Wang MD, Fojo AT, Sunshine M, Narasimhan S, Kane DW, Reinhold WC, Lababidi S et al. 2003 GoMiner: a resource for biological interpretation of genomic and proteomic data. Genome Biology 4 R28. (https://doi.org/10.1186/gb-2003-4-4-r28)
Zhang J, Tu Q, Bonewald LF, He X, Stein G, Lian J \& Chen J 2011 Effects of miR-335-5p in modulating osteogenic differentiation by specifically downregulating Wnt antagonist DKK1. Journal of Bone and Mineral Research 26 1953-1963. (https://doi.org/10.1002/jbmr.377)

Zhang K, Zhong W, Li WP, Chen ZJ \& Zhang C 2017a miR-15a-5p levels correlate with poor ovarian response in human follicular fluid. Reproduction 154 483-496. (https://doi.org/10.1530/REP-17-0157)

Zhang LL, Zhang LF, Guo XH, Zhang DZ, Yang F \& Fan YY 2017b Downregulation of miR-335-5p by long noncoding RNA ZEB1-AS1 in gastric cancer promotes tumor proliferation and invasion. DNA and Cell Biology 37 46-52. (https://doi.org/10.1089/dna.2017.3926)

Received 5 May 2018

First decision 13 June 2018

Revised manuscript received 13 August 2018

Accepted 4 September 2018 